\title{
NUMERICAL SIMULATIONS OF COLLAPSE OF A MAGNETIZED ROTATING MOLECULAR CLOUD
}

\author{
KANJI OHTA AND ASAO HABE \\ Department of Physics, Hokkaido University, Sapporo, 060 JAPAN
}

\section{INTRODUCTION}

Recent observations reveal the velocity structure of star forming regions and the magnetic field in molecular clouds. It is known from observations that the molecular clouds rotate. It is suggested that the magnetic field have a important roll of the star formation process(e.g. Myers and Goodman 1988) and rotation of cloud have effects for evolution of molecular cloud. However it is not cleared how the magnetic field plays a roll of the star formation process in a rotating clond.

In the previous theoretical studies, most of simulations are performed for collapse process of a rotating cloud without magnetic field (e.g. Miyama et al. 1984, Boss 1990) or collapse process of a magnetized cloud without rotation (e.g. Scott and Black 1980). Dorfi(1982) studied collapse of a magnetized, rotating cloud. However he did not calculate those with high resolutions, since he performed 3-dimensional calculations of about 6000 grid points.

Since observation instruments have been developed, it is possible to observe the star forming regions with good resolution. We study the collapse of the rotating, magnetized, isothermal cloud by mean of the axisymmetric numerical simulations with high resolution.

\section{INITIAL CONDITIONS AND NUMERICAL METHODS}

we assumed axisymmetry to the rotation axis for simplicity. We adopted cylindrical coordinates in which the $\mathrm{Z}$-axis is the rotation axis.

We assume that the molecular cloud is initially an isothermal gas sphere with rigid rotation and uniform density, $\rho_{o}$. And we assume that ambient gas is initially rest and pressure equilibrium with the cloud gas but $\rho_{o, a m b}=0.1 \rho_{o}$ where $\rho_{o, a m b}$ is the initial density of ambient gas. We also assume that the magnetic field is uniform, parallel to rotation axis and frozen in the gas. Then we can describe gas motion by the magnetohydrodynamics (MHD).

There are three parameters that divide the dynamical state of the rotating magnetized isothermal cloud, defined as $\alpha=$ thermal energy/|gravitational energy |, $\beta=$ rotational energy/| gravitational energy $\mid, \gamma=$ magnetic energy/|gravitational energy $\mid$. We have calculated four cases, that are with same $\alpha(=0.3), \beta(=0.1)$, but different $\gamma(=0.0,0.05,0.2,0.5)$.

We used axisymmetric smoothed particle hydrodynamics (SPH) for calculating the self gravitating gas motion and second order cell method for solving the Lorentz force and the induction equation. We carried out our simulation 
using about $N=7000$ particles. We used cell in order to calculate Lorentz force and solve the induction equation. The mesh numbers are $100 \times 200$. We solved the self-gravity by "vectorized " successive line over-relaxation(SLOR) method.

\section{RESULTS}

Our numerical results show that, after the magnetized rotating molecular cloud contracts(Figure 1-a, b) by self gravity, high density disk is formed(Figure 1-c). In central region of the disk, the magnetic field transfers the angular momentum from the disk to ambient gas by the torsional Alfven wave, even if initially the magnetic field energy density is much smaller than the gravitational energy density of the molecular cloud. Then the central region of the high density disk changes into the core structure(Figure 1-d). On the other hand, the high density ring is formed in the result of the collapse of same clond without magnetic field. These results indicate that the magnetic field prevents the molecular cloud from fragmentation.

\section{REFERENCES}

Boss, A. P. 1990, in Physical Processes in Fragmentation and Star Formation, Capuzzo-Dolcetta, R., Chiosi, C. and Fazio, A., (Kluwer Academic Pub.), 279.

Dorfi, E. 1982, $A B A, 114,151$.

Miyama, S. M., Hayashi, C. and Narita, S., 1984, ApJ, 279, 621.

Myers, P. C.and Goodman, A. A., 1988, ApJ, 329, 392.

Scott, E. and Black, D. C. 1980, ApJ, 239, 166.
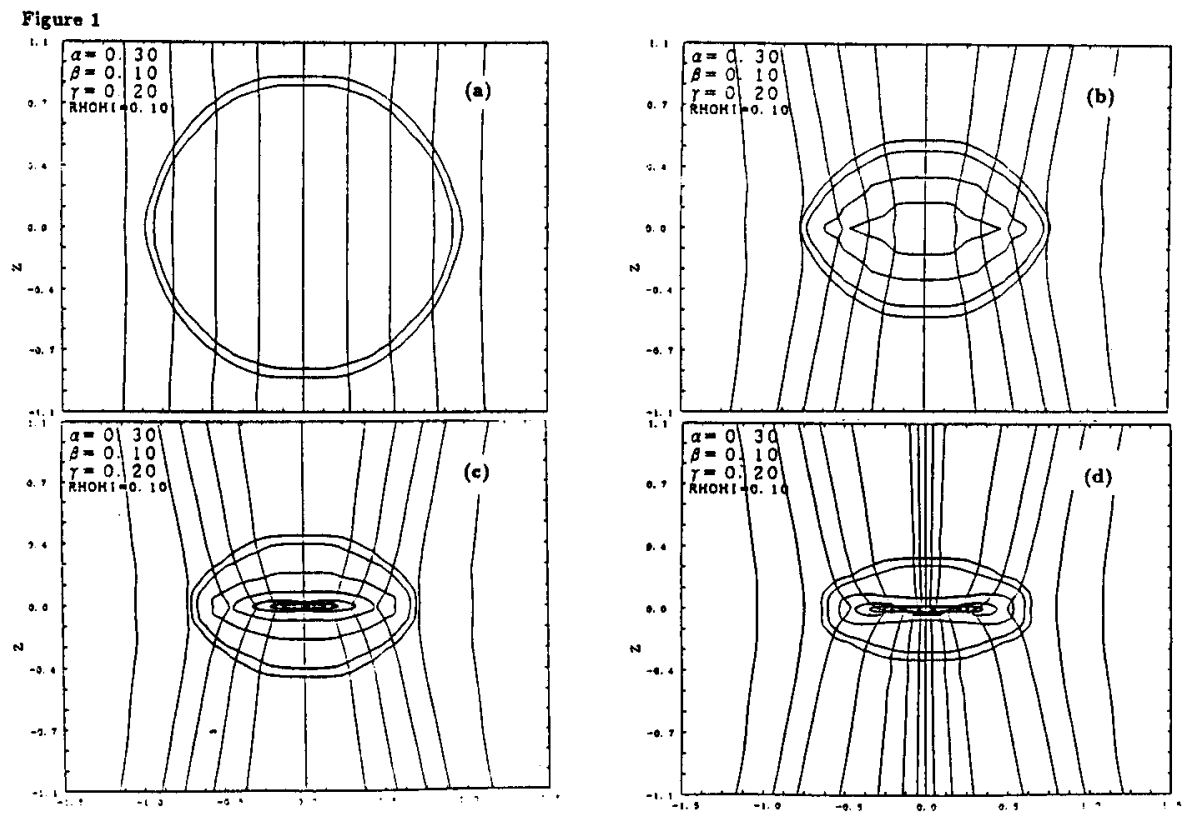